\title{
A scattering model for nano-textured interfaces and its application in opto-electrical simulations of thin-film silicon solar cells
}

\author{
K. Jäger, ${ }^{\text {a),b) }}$ M. Fischer, ${ }^{\text {b) }}$ R. A. C. M. M. van Swaaij, ${ }^{\text {b) }}$ and M. Zeman ${ }^{\text {) }}$ \\ Photovoltaic Materials and Devices Laboratory—DIMES, Delft University of Technology, P.O. Box 5031, \\ 2600 GA Delft, The Netherlands
}

(Received 10 January 2012; accepted 17 March 2012; published online 23 April 2012)

\begin{abstract}
We present a scattering model based on the scalar scattering theory that allows estimating far field scattering properties in both transmission and reflection for nano-textured interfaces. We first discuss the theoretical formulation of the scattering model and validate it for nano-textures with different morphologies. Second, we combine the scattering model with the opto-electric ASA simulation software and evaluate this combination by simulating and measuring the external parameters and the external quantum efficiency of solar cells with different interface morphologies. This validation shows that the scattering model is able to predict the influence of nano-textured interfaces on the solar cell performance. The scattering model presented in this manuscript can support designing nano-textured interfaces with optimized morphologies. (c) 2012 American Institute of Physics. [http://dx.doi.org/10.1063/1.4704372]
\end{abstract}

\section{INTRODUCTION}

Due to less energy and material consumption during production, thin-film silicon solar-cell technology is an environmental friendly alternative to crystalline silicon technology. In order to maximize the short circuit current density, one of the major aims is to maximize the absorption of the photons in the absorber layer. At the same time, the absorber layer is kept as thin as possible in order to minimize electron-hole recombination and to keep light-induced degradation at a low level. ${ }^{1}$

To obtain high short-circuit current densities in thin absorber layers, light trapping techniques are generally applied. In addition to optimized back reflectors, scattering at randomly nano-textured interfaces is the most commonly used technique. ${ }^{2,3}$ In $\mathrm{p}$-i-n thin-film silicon solar cells, the nano textures are created by depositing the electrically active layers on a glass superstrate that is coated with a surface-textured transparent conductive oxide (TCO) layer. Due to scattering at the rough interfaces, the average photon path length in the absorber layer is increased, leading to a higher absorption.

Figure 1 illustrates superstrate thin-film silicon solar cells with flat interfaces (a) and with randomly nanotextured interfaces (b). In order to maximize the absorption, the surface morphology of the rough interfaces has to be optimized, e.g., by modeling. Hence, an accurate scattering model is required that relates the interface morphology to the scattering parameters. In the last two years, several models were published that can predict the angular intensity distribution (AID) and haze in transmission. ${ }^{4-6}$ While the details of the models differ, their main principle is the same: the scattered field is related to the scattering object via Fourier transforms. Since these models take the whole

\footnotetext{
${ }^{a)}$ Electronic mail: k.jaeger@tudelft.nl.

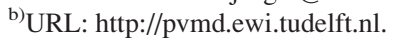

morphology of the scattering interface into account, they are superior to older models that use as input only the rmsroughness of the interface. ${ }^{7-10}$

The main purpose of this work is to present and discuss an extended scattering model that is valid for both transmission and reflection and can be applied for interfaces between arbitrary materials. To find the correct formulation for the scattering model, we revisit and improve the different models on transmission. To evaluate the model, it is not sufficient to study the scattering properties of TCO-air interfaces. We mainly have to consider interfaces between materials that are present in the solar cells. We therefore use recent results from Schulte et al., ${ }^{11}$ who validated different scattering models by comparing the simulated AID of a nano-textured TCO-silicon interface with measured data. Similar as Schulte et al. did for transmission, we also study the AID in reflection for interfaces between zinc oxide and different materials.

The second emphasis of this article is to show that the scattering model combined with an opto-electrical device simulator can be used to study the effect of nano-textured interfaces on the solar cell performance. We discuss optoelectrical simulations of thin-film silicon solar cells with nano-textured interfaces for three different morphologies. We then compare the simulated $J-V$ characteristics and the external quantum efficiencies with measured data.

\section{THE SCATTERING MODEL AND ITS EVALUATION}

Due to increasing computer capacity, rigorous solvers of the Maxwell equations, like the finite difference time domain (FDTD) method ${ }^{12,13}$ or the finite elements method, ${ }^{14}$ have become very popular in recent years. Nonetheless, the scalar scattering theory still is a very powerful instrument for estimating the scattering properties of nano-textured interfaces. The main tool of the scalar scattering theory is diffraction integrals, such as the Fresnel-Kirchhoff diffraction formula, 

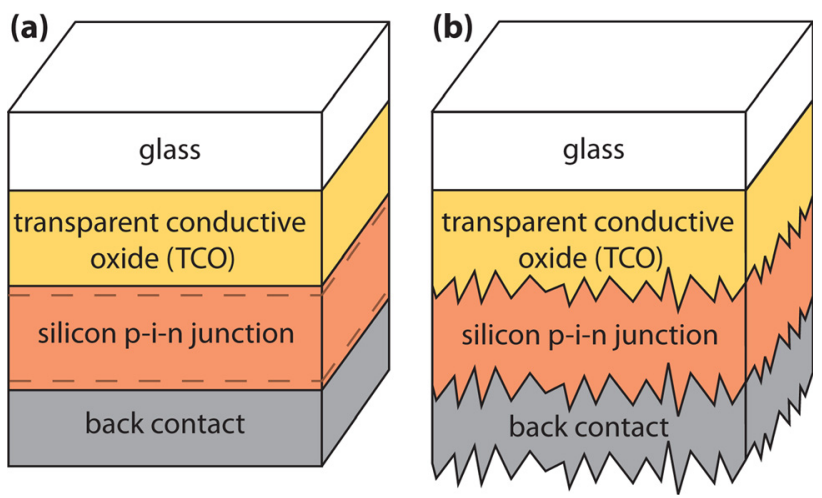

FIG. 1. Illustrating thin-film silicon solar cells with flat interfaces (a) and with nano-textured interfaces (b) in p-i-n configuration. (Layer thicknesses not in scale.)

from which the Fraunhofer and Fresnel diffraction integrals can be deduced as first order and second order approximations, respectively, and the Rayleigh-Sommerfeld diffraction integrals. ${ }^{15}$

In this section, we first present the scattering model. We then validate the model on TCO-air interfaces and finally on interfaces between materials that are used in solar cells.

\section{A. The model}

In the scalar scattering theory, light is described as a complex scalar field $U(\mathbf{r})$ instead of the electromagnetic fields $\mathbf{E}(\mathbf{r})$ and $\mathbf{B}(\mathbf{r}) .{ }^{15}$ In the model, we formulate the equations such that energy conservation is intrinsically ensured. We determine the scattering properties in a two-step process. First, we determine the scattered field in a two-dimensional $k$-space. Second, we relate this field to the angular intensity distribution and the haze. Our approach is strongly related to the Fraunhofer diffraction integral. However, there are important differences:

1. The classical Fraunhofer integral contains a paraxial approximation making it less accurate for larger scattering angles. In the second step of our model, we circumvent the need for a paraxial approximation by relating the field in $k$-space to the AID rigorously. This is similar to the approach presented by Harvey et al. ${ }^{16}$

2. In classical diffraction theory, light is diffracted at an aperture. Mathematically this is done by defining pupil functions $G$ that take a constant value inside the aperture and vanish outside. In our model, we consider the area in which the interface morphology is known, as aperture. In difference to the classical case, our pupil functions are not constant inside the aperture, but contain a phase shift that depends on the height profile of the interface.

In detail, the field in $k$-space is given by the twodimensional Fourier transform of the pupil functions $G_{T}$ and $G_{R}$

$$
\begin{aligned}
& U_{T}\left(K_{x}, K_{y}\right)=\frac{1}{2 \pi} \iint_{\mathbb{R}^{2}} G_{T}(x, y) e^{-i\left(K_{x} x+K_{y} y\right)} \mathrm{d} x \mathrm{~d} y, \\
& U_{R}\left(K_{x}, K_{y}\right)=\frac{1}{2 \pi} \iint_{\mathbb{R}^{2}} G_{R}(x, y) e^{-i\left(K_{x} x+K_{y} y\right)} \mathrm{d} x \mathrm{~d} y .
\end{aligned}
$$

The subscripts $T$ and $R$ denote transmittance and reflectance, respectively. The pupil functions are given by

$$
\begin{aligned}
G_{T}(x, y) & =\sqrt{\frac{T}{A}} \exp \left[i k_{0} z(x, y)\left(n_{1}-n_{2}\right)\right], \\
G_{R}(x, y) & =\sqrt{\frac{R}{A}} \exp \left[i k_{0} z(x, y) 2 n_{1}\right]
\end{aligned}
$$

inside the aperture while outside the aperture $G_{T, R} \equiv 0$. Here, $k_{0}=2 \pi / \lambda_{0}$ denotes the wavenumber in vacuo, where $\lambda_{0}$ is the wavelength. The interface is formed between two materials with the refractive indices $n_{1}$ and $n_{2}$. The light is incident on the rough interface from the material with $n_{1}$. The morphology of the interface is contained in the height function $z(x, y)$. The constants $\sqrt{T / A}$ and $\sqrt{R / A}$ are chosen such that the total amount of light flowing through the aperture of area $A$ is equal to the total transmittance $T$ or the total reflectance $R$ of the interface, respectively. Hence, $T$ and $R$ are input parameters of the scattering model. We note that in $G_{T}$ both refractive indices are present in the exponent, while in $G_{R}$ only $n_{1}$ is present in the exponent. This indicates that the shape of the $\mathrm{AID}_{R}$ is independent of $n_{2}$, while its strength is controlled by $R$. We will show below that this assumption is supported by measurements. The Plancherel theorem ensures energy conservation through the Fourier transform ${ }^{17}$

$$
\iint_{\mathbb{R}^{2}}\left|G_{T, R}\right|^{2} \mathrm{~d} x \mathrm{~d} y=\iint_{\mathbb{R}^{2}}\left|U_{T, R}\right|^{2} \mathrm{~d} K_{x} \mathrm{~d} K_{y}=T, R .
$$

Only the $k$-vectors inside a circle with radius $k_{0} n_{2,1}, K_{x}^{2}$ $+K_{y}^{2}<k_{0}^{2} n_{2,1}^{2}$ correspond to transmitted (reflected) light, while $k$-vectors outside this circle correspond to evanescent waves. The subscripts 2 and 1 are used for transmission and reflectance, respectively. Therefore, all the power will be transmitted (reflected) from within this circle. As Harvey et $a l .{ }^{16}$ pointed out, we therefore have to redistribute the energy by multiplying the field $U_{T, R}$ inside the circle with the factor

$$
\kappa_{T, R}=\frac{\iint_{\mathbb{R}^{2}}\left|U_{T, R}\right|^{2} \mathrm{~d} K_{x} \mathrm{~d} K_{y}}{\iint_{\bigcirc}\left|U_{T, R}\right|^{2} \mathrm{~d} K_{x} \mathrm{~d} K_{y}},
$$

while $U_{T, R}\left(K_{x}, K_{y}\right) \stackrel{!}{=} 0$ if $K_{x}^{2}+K_{y}^{2}>k_{0}^{2} n_{2,1}^{2}$. The $\bigcirc$ denotes the set defined by $K_{x}^{2}+K_{y}^{2} \leq k_{0}^{2} n_{2,1}^{2}$.

The intensity and hence the AID is related to $|U|^{2}$. As illustrated in Fig. 2, the AID is defined on a unit hemisphere with coordinates $(\theta, \phi)$, while $U$ lives on the flat $k$-space with coordinates $\left(K_{x}, K_{y}\right)$. Instead of using the paraxial approximation, we use the accurate coordinate transformation, in which the coordinates are related to each other via $K_{x}=k_{0} n_{2,1} \sin \theta \cos \phi$ and $K_{y}=k_{0} n_{2,1} \sin \theta \sin \phi$. The AID then is given by

$$
\operatorname{AID}_{T, R}(\theta, \phi)=k_{0} n_{2,1}\left|U_{T, R}\right|^{2} \cdot \cos \theta .
$$

If the morphology of the nano-textured interface is nearly isotropic, $U$ is isotropic as well. This implies that, up to noise due to limited sampling size of the interface, the AID is independent 

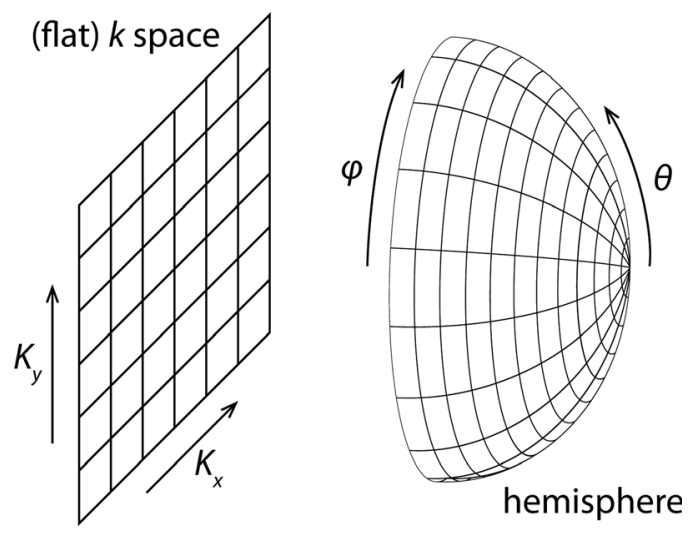

FIG. 2. Illustrating the flat $k$-space on which $U\left(K_{x}, K_{y}\right)$ lives and the hemisphere, on which the AID is defined.

of $\phi$. We can therefore reduce the noise by averaging the AID over circles with constant $\theta$

$$
\operatorname{AID}_{T, R}(\theta)=\frac{k_{0} n_{2,1}}{2 \pi \sin \theta} \oint_{C}\left|U_{T, R}\right|^{2} \mathrm{~d} s \cdot \cos \theta .
$$

The line integral is taken along the circle $C$ that is defined by the condition $K_{x}^{2}+K_{y}^{2}=k_{0}^{2} n_{2,1}^{2} \sin ^{2} \theta$. The cosine arises from the coordinate transformation between the flat $k$-space and the curved hemisphere on which the AID is defined.

In general, the height function $z(x, y)$ is not available everywhere in the aperture, but only at a finite number of points that, for example, are obtained with atomic force microscopy (AFM). Hence, we will use discrete (fast) Fourier transforms instead of continuous Fourier transforms. The constants in the pupil functions from Eq. (3) will then be $\sqrt{T / N}$ and $\sqrt{R / N}$ instead of $\sqrt{T / A}$ and $\sqrt{R / A}$, where $N$ is the number of data points. Further, the integrals have to be replaced by sums.

The haze in transmission (reflection) is defined as the ratio of the diffuse transmittance (reflectance) to the total transmittance (reflectance), $H_{T, R}=Q_{\text {dif }} / Q_{\text {tot }}$, where $Q$ denotes either $T$ or $R$. In the discrete case, where $z(x, y)$ is given as a discrete set of points, the haze can be estimated in a straightforward manner by

$$
\begin{aligned}
& H_{T, R}(\lambda)=\frac{Q_{\text {dif }}(\lambda)}{Q_{\text {tot }}(\lambda)}=\frac{Q_{\text {tot }}(\lambda)-Q_{\text {spec }}(\lambda)}{Q_{\text {tot }}(\lambda)} \\
& =\frac{\sum_{K_{x}^{2}+K_{y}^{2} \leq k_{0}^{2} n_{2,1}^{2}}\left|U_{T, R}\left(K_{x}, K_{y}\right)\right|^{2}-\left|U_{T, R}(0,0)\right|^{2}}{\sum_{K_{x}^{2}+K_{y}^{2} \leq k_{0}^{2} n_{2,1}^{2}}\left|U_{T, R}\left(K_{x}, K_{y}\right)\right|^{2}}
\end{aligned}
$$

as Dominé et al. demonstrated. ${ }^{5}$ In difference to the AID, the haze is affected by neither the Harvey normalization nor $R$ or $T$. The wavelength dependent changes of the haze therefore are mainly due to the changing wavenumber $k_{0}=2 \pi / \lambda_{0}$. In this model, the haze therefore in general will decrease with increasing wavelength, given that the refractive index does not change too fast with the wavelength.

\section{B. Discussion on the choice of $G_{T}$}

Since in the last years several models for transmission have been presented, we want to discuss the choice of the pupil function for transmission, before we proceed with the experimental evaluation of the model.

Jäger and Zeman developed a model based on first order Born approximation. ${ }^{4}$ At first, only scattering of objects in vacuo can be handled in that model. To calculate scattering parameters between arbitrary materials, the surrounding vacuum has to be replaced with a material of refractive index $n_{2}$. This can be done by performing the transformations $n_{1} \rightarrow n_{1}^{\text {eff }}$ $=n_{1} / n_{2}$ and $k_{0} \rightarrow k_{\text {eff }}=k_{0} n_{2}$. Then, the shape of the scattered field is determined by the pupil function $G_{T}^{B}=\exp \left[i k_{\mathrm{eff}} z(x, y)\right]$ $=\exp \left[i k_{0} z(x, y) n_{2}\right]$. The strength of the scattered field is controlled by the so-called scattering potential, which vanishes if $n_{1} \rightarrow n_{2}$.

Bittkau et al. formulated the grating model in which the scattering surface is decomposed into a superposition of gratings. ${ }^{6}$ The scattering angles of these gratings are determined by their reciprocal lattice constants. Finally, Dominé et al. developed a scattering model based on the RayleighSommerfeld diffraction integral ending up with the pupil function used in Eq. (3). ${ }^{5}$

All three models predict the scattering properties of TCO-air interfaces well. For the model formulated by Jäger and Zeman and that developed by Dominé et al., this can be understood easily by setting the refractive index of the TCO to $n_{1}=2$. Then, the two pupil functions become similar: $G_{T}^{B}=\exp \left[i k_{0} z(x, y) \cdot 1\right]=\exp \left[i k_{0} z(x, y)\right]$ and $G_{T}=$ $\exp \left[i k_{0} z(x, y)(2-1)\right]=\exp \left[i k_{0} z(x, y)\right]$. However, the models behave very differently when applied to TCO-silicon interfaces, as was discussed in a recent publication by Schulte et al.: ${ }^{11}$ While both the grating model and the model based on the Rayleigh-Sommerfeld integral estimate the measured values well, the model based on the first order Born approximation overestimates the scattered field. This indicates that the assumption for $G_{T}^{B}$, namely that only the phase obtained in the second medium influences the shape of the scattered field, is not met in this case. The grating model is insensitive to stretching the vertical features of the scattering interface. Even though it predicts the scattered field well for the investigated samples, the general predictive power of this model is unclear at this moment. Hence, using the pupil function as formulated by Dominé et al. currently is the method of choice.

\section{Experimental evaluation on TCO-air interfaces}

Typically rough interfaces are introduced into the solar cells by using nano-textured TCO layers as substrates. We used four different TCO materials on glass to evaluate the model: one sample of fluorine-doped tin oxide $\left(\mathrm{SnO}_{2}: \mathrm{F}\right)$ of Asahi U-type, ${ }^{18}$ two samples of sputtered, aluminium-doped zinc oxide ( $\mathrm{ZnO}: \mathrm{Al})$ that was etched in a $0.5 \% \mathrm{HCl}$ solution, ${ }^{3,19}$ and one sample of boron-doped LP-CVD zinc-oxide ( $\mathrm{ZnO}: \mathrm{B})$ of so-called "B-type" from PV-LAB of the École polytechnique fédérale de Lausanne (EPFL), Switzerland. ${ }^{20}$

The morphology, rms roughness $\sigma_{r}$ and correlation length $\ell_{c}$ of the four samples are summarized in Table I. To determine these parameters, we performed AFM scans of $256 \times 256$ points over an area of $20 \times 20 \mu \mathrm{m}^{2}$ with a NT-MDT NTEGRA Aura AFM in tapping mode. Because of the small lateral feature size of $\mathrm{SnO}_{2}: \mathrm{F}$ we also included $\sigma_{r}$ and $\ell_{c}$ derived from 
TABLE I. The morphology, rms roughness $\sigma_{r}$ and correlation length $\ell_{c}$ of the used TCO samples as obtained from AFM scans of $256 \times 256$ points over $20 \times 20 \mu \mathrm{m}^{2}$. The values in brackets show values obtained from $5 \times 5 \mu \mathrm{m}^{2}$ scans.

\begin{tabular}{lccc}
\hline \hline Material & Morphology & $\sigma_{r}(\mathrm{~nm})$ & $\ell_{c}(\mathrm{~nm})$ \\
\hline $\mathrm{SnO}_{2}: \mathrm{F}$ (Ref. 18) & Pyramidal & $40(37)$ & $175(160)$ \\
$\mathrm{ZnO}: \mathrm{Al}$ (Refs. 3 and 19) & Crater-like & 60 & 520 \\
$\mathrm{ZnO}: \mathrm{Al}$ & Crater-like & 90 & 625 \\
$\mathrm{ZnO}: \mathrm{B}$ (Ref. 20) & Pyramidal & 220 & 470 \\
\hline \hline
\end{tabular}

$5 \times 5 \mu \mathrm{m}^{2}$ scans. We used gold coated silicon probes of type NT-MDT NSG 10. From these scans, we obtained the height function $z(x, y)$ by subtracting the average plane and extracted $\sigma_{r}$ and $\ell_{c}$ by applying Gaussian fits on the auto-correlation function and the height-height correlation function of $z(x, y){ }^{21}$ For $\mathrm{SnO}_{2}: \mathrm{F}$ and $\mathrm{ZnO}: \mathrm{Al}$, we determined the $n, k$ data by the method described by Sap et al. ${ }^{23}$ The $n, k$ data of $\mathrm{ZnO}: \mathrm{B}$ were provided by the PV-LAB of the EPFL.

For the evaluation of the model, we approximated $R$ and $T$ by using the Fresnel equations for flat interfaces at normal incidence. While for transmission the light was incident on the glass side, for reflection it was incident on the coating side. This was done to ensure that light reached the detector without traversing any other interfaces after being scattered. Although results on transmission were already discussed several times, we will also show them here for the sake of completeness.

Figure 3 shows the simulated and measured AID in transmission (a) and reflection (b). Here, the simulated AIDs were smoothened. Up the $60^{\circ}$, we observe good agreement for $\mathrm{SnO}_{2}: \mathrm{F}$ and $\mathrm{ZnO}: \mathrm{Al}$. For the AID for angles higher than $60^{\circ}$, the deviations become larger. However, also the measurement uncertainty becomes larger for these low intensities. ${ }^{22}$ For $\mathrm{ZnO}: \mathrm{B}$, the simulated $\mathrm{AID}_{T}$ overestimates the measured data, while the simulated $\mathrm{AID}_{R}$ underestimates the measured data. These deviations are due to the high roughness of the $\mathrm{ZnO}: \mathrm{B}$ sample, letting $R$ and $T$ deviate significantly from the values expected from the Fresnel equations. While rough interfaces usually have an anti-reflective (AR) effect that, for example, can be modeled with effective medium approaches, the investigated $\mathrm{ZnO}: \mathrm{B}$ sample behaves contrarily: It transmits less and reflects more than $\mathrm{ZnO}: \mathrm{B}$ samples with a lower roughness, as we have confirmed with measurements with an integrating sphere. Despite this inaccuracy in the determination of $R$ and $T$, the shape of the AID is predicted well also for the very rough $\mathrm{ZnO}: \mathrm{B}$ sample.

Figure 4 shows the simulated and measured haze in transmission (a) and reflection (b). We observe good agreement between measurements and simulations. The calculated haze in reflection does not show the interference fringes, since the model only covers the physics at the interface but does not contain thin-film optics, which is not within the scope of this model.

\section{Experimental evaluation on interfaces between TCO and different materials}

As already mentioned, solar cells do not contain TCOair interfaces but interfaces between, e.g., TCO and silicon or silicon and metal. Therefore, it is very important to test

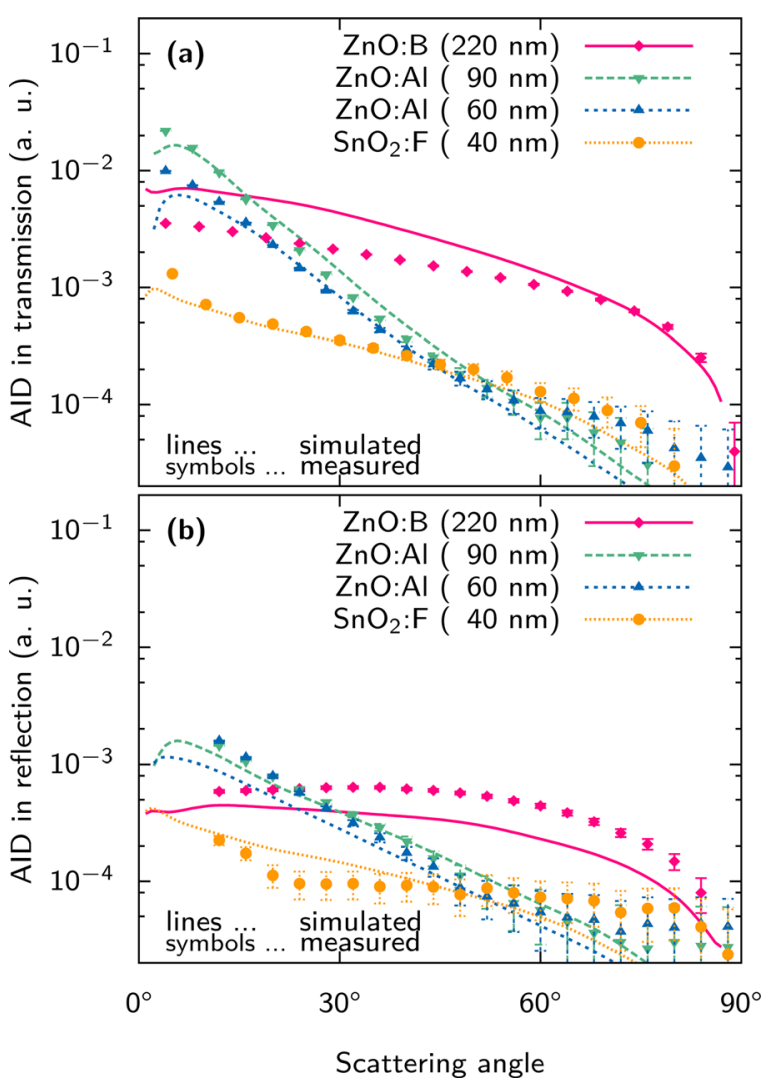

FIG. 3. Simulated and measured AID in transmission (a) and reflection (b) for four TCO-air interfaces with different morphologies at $600 \mathrm{~nm}$. The measurement deviations are determined according to Ref. 22.

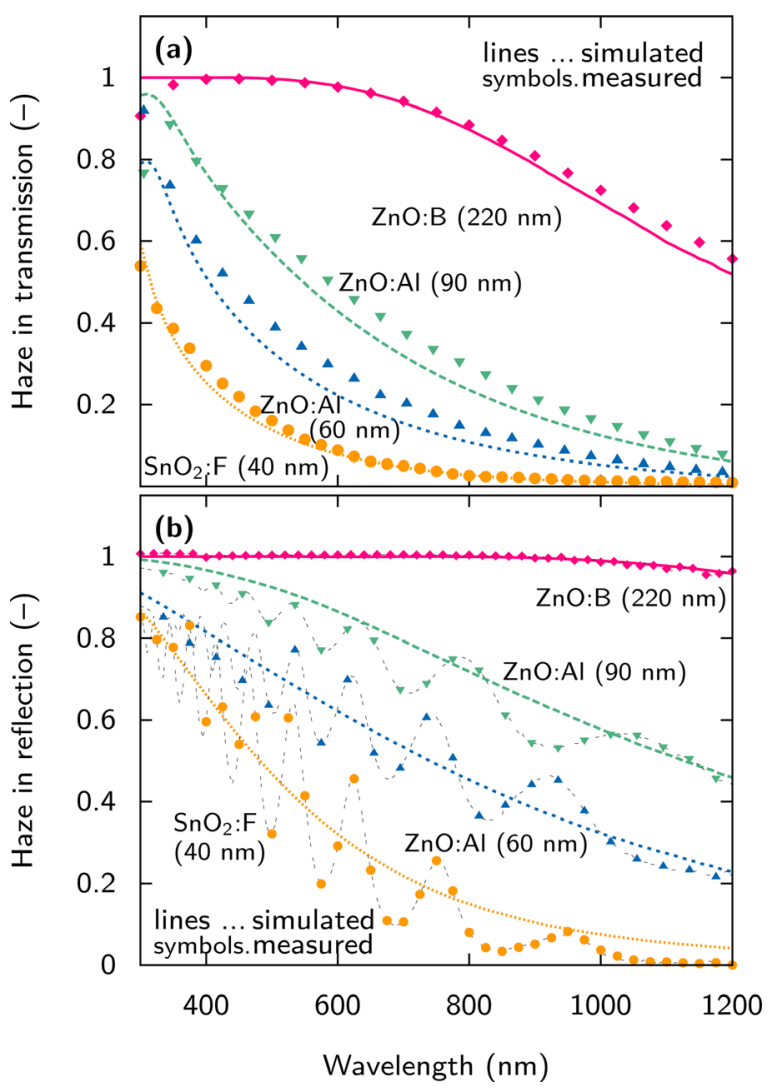

FIG. 4. Simulated and measured haze in reflection in transmission (a) and reflection (b) for four TCO-air interfaces with different morphologies. 


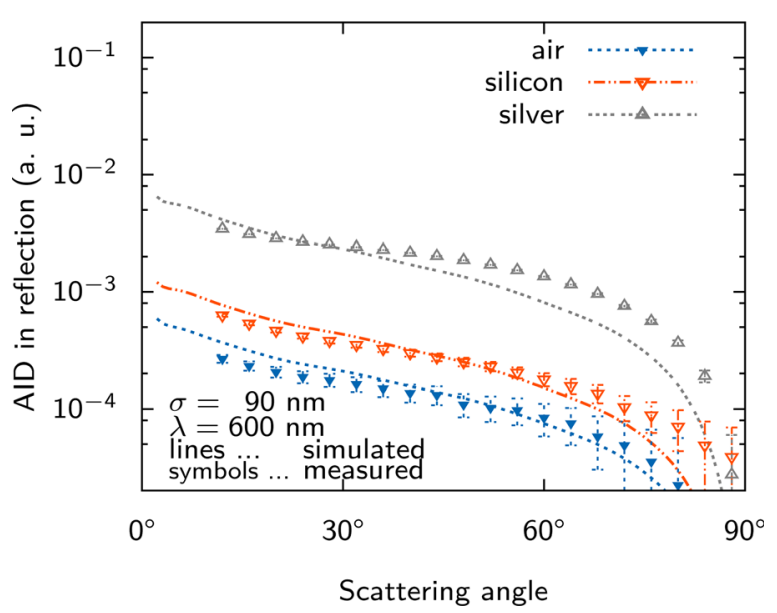

FIG. 5. Simulated and measured angular intensity distribution in reflection for three different $\mathrm{ZnO}$ :Al-material interfaces. The measurement deviations are determined according to Ref. 22.

the model also on these interfaces. Since results for the $\mathrm{AID}_{T}$ at TCO-silicon interfaces were recently published by Schulte et al. ${ }^{11}$, we will now focus on the $\mathrm{AID}_{R}$ at interfaces between TCO and different materials.

We prepared three samples of etched $\mathrm{ZnO}$ :Al with $\sigma_{r} \approx$ $90 \mathrm{~nm}$ and $\ell_{c} \approx 625 \mathrm{~nm}$. We left the first sample as it was, deposited a $1 \mu \mathrm{m}$ thick layer of amorphous hydrogenated silicon $(\mathrm{a}-\mathrm{Si}: \mathrm{H})$ on the second sample and deposited $300 \mathrm{~nm}$ of silver on the third sample.

In difference to the results on reflection presented in Fig. 4(b), here we had to measure from the glass side. Before the scattered light is measured, it therefore passes the $\mathrm{ZnO}$ :Al-glass and the glass-air interface. To simulate the measured AID, we thus had to take several effects into account similar to the effects discussed by Schulte $e t$ al.

We first corrected for the transmission at the $\mathrm{ZnO}: \mathrm{Al}-$ glass and the glass-air interface with the Fresnel equations. Second, we corrected the angles with Snell's law. Third, we had to consider that the detector does not detect a single ray but a pencil of rays that covers a certain solid angle. After passing through a (flat) interface, this pencil has a different solid opening angle. Its étendue, however, is conserved. ${ }^{24}$ We thus obtain the factor $n_{\mathrm{TCO}}^{-2} \cdot \cos \theta_{v a c} / \cos \theta_{\mathrm{TCO}}$.

Schulte et al. did not compare the models directly to the measurements but compared them to results obtained with the FDTD method. They did this because the models assume interfaces between two halfspaces. The measured values, however, also contain light that is reflected back into the sample at the Si-air interface and then reflected and scattered again at the rough $\mathrm{ZnO}: \mathrm{Al}-\mathrm{Si}$ interface, and higher orders. In principle, the same is true here, but the secondary interfaces in our experiment are much less reflective: While the reflectance of the Si-air interface investigated by Schulte et al. was $33.2 \%$ at $600 \mathrm{~nm}$ and normal incidence, the reflectance of the $\mathrm{ZnO}$ :Al-glass and glass-air interfaces is only $0.8 \%$ and $4.1 \%$, respectively. It is therefore a good approximation to neglect these higher order effects, even though one then can expect larger deviations at higher angles, where the reflectivity is higher. Furthermore, we can neglect secondary reflections from the Si-air interface of the second sample since at $600 \mathrm{~nm}$ only about $0.1 \%$ of light that is transmitted into the silicon will reach the $\mathrm{ZnO}: \mathrm{Al}$ interface again after it is reflected at the Si-air interface.

Figure 5 shows the measured and simulated $\mathrm{AID}_{R}$ at $\mathrm{ZnO}: \mathrm{Al}$-air, $\mathrm{ZnO}: \mathrm{Al}-\mathrm{Si}$, and $\mathrm{ZnO}$ :Al-silver interfaces. Like in Fig. 3, the simulated AIDs were smoothened. As we can see, the agreement in general is good, and the model predicts the material-dependent changes of the $\mathrm{AID}_{R}$ correctly. The deviations at larger angles are indeed higher what can be expected from the discussion above. In this evaluation, we have discussed the $\mathrm{AID}_{R}$ of interfaces between TCO and different materials. The $\mathrm{AID}_{R}$ within $\mathrm{Si}$, e.g., at $\mathrm{Si}-\mathrm{TCO}$ or $\mathrm{Si}-$ metal interfaces has not been evaluated in this work and remains an important topic for future research.

\section{SIMULATING WHOLE SOLAR CELL STRUCTURES}

In this section, we combine the scattering model with the ASA opto-electric device simulator that has been developed at the Delft University of Technology. ${ }^{25}$ This allows us to study the effect of different nano-textured interfaces onto the external solar cell parameters. The section is divided into two subsections: In the subsection III A, we discuss the experimental solar cell series that was prepared for the validation of the simulations. In the subsection III B, we discuss opto-electric simulations of cells with different nanotextured interface morphologies and validate the obtained results by comparing them with measured data.

\section{A. Experimental solar cell series}

The scattering model is developed in the far field regime, i.e., it predicts the field at distances that are at least several wavelengths away from the scattering object. State-of-the-art thin-film silicon solar cells are deposited with an i-layer thickness between 250 and $300 \mathrm{~nm}$. Taking the real part of the refractive index of amorphous silicon to be approximately 4, light with $600 \mathrm{~nm}$ vacuum wavelength inside silicon has a wavelength of about $150 \mathrm{~nm}$. Having the light being scattered at both sides of the i-layer, near field effects, i.e., at subwavelength scales, might become important, ${ }^{26}$ making the use of the scattering model arguable. We therefore decided to prepare solar cells with an amorphous intrinsic layer with a thickness of approximately $700 \mathrm{~nm}$. At this thickness, the application of the far field approach is more justified.

Figure 6 illustrates the detailed structure of the prepared solar cells with the nominal layer thicknesses $d$. As TCO we used RF magnetron sputtered $\mathrm{ZnO}$ :Al. To texture the surface, the zinc oxide was etched in hydrochloric acid with $0.5 \% \mathrm{HCl}$ mass fraction after sputtering. ${ }^{3} \mathrm{We}$ varied the etching time between $0 \mathrm{~s}$ and $75 \mathrm{~s}$ with $15 \mathrm{~s}$ steps in between. To minimize the effect of changing TCO thickness on its transmittance and sheet resistance, we varied the deposition time and hence the initial TCO thickness. This was done in order to keep the final average TCO thickness after etching approximately constant.

The top panels of Fig. 7 show AFM scans of $5 \times 5 \mu \mathrm{m}^{2}$ for the $\mathrm{ZnO}: \mathrm{Al}$ after $15 \mathrm{~s}, 30 \mathrm{~s}$, and $45 \mathrm{~s}$ etching time. The morphology shows the typical crater like features of etched $\mathrm{ZnO}: \mathrm{Al}$; the rms roughness is $35 \mathrm{~nm}, 70 \mathrm{~nm}$, and $95 \mathrm{~nm}$, 


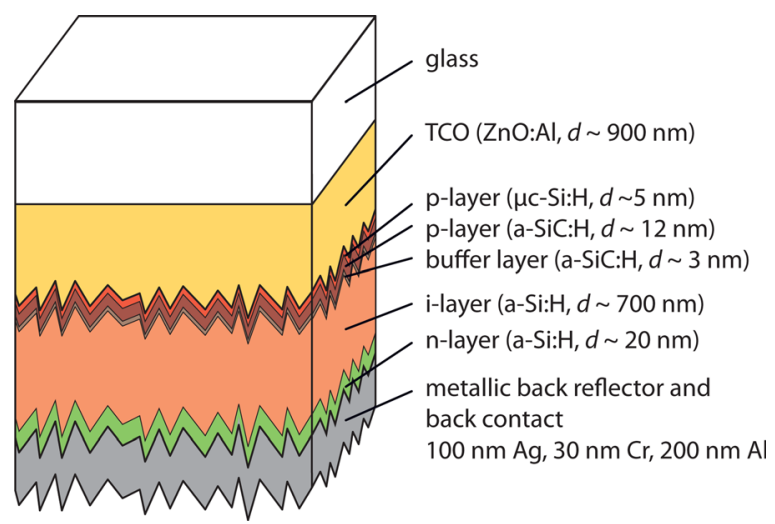

FIG. 6. Illustrating thin-film silicon solar cells as they were deposited for this contribution. The layer thickness is denoted by $d$. (Layer thicknesses not in scale.)

respectively. The bottom panels show the back of p-i-n structures with an i-layer thickness of $700 \mathrm{~nm}$ that were deposited on the back of these $\mathrm{ZnO}$ :Al layers (see below). The morphology is smoother than of the $\mathrm{ZnO}: \mathrm{Al}$ interfaces resulting in a lower rms roughness of about $15 \mathrm{~nm}, 50 \mathrm{~nm}$, and $85 \mathrm{~nm}$, respectively. The AFM scans were obtained with the instrument discussed in Sec. II C. Figure 8 shows the RMS roughness and the correlation length of the $\mathrm{ZnO}$ :Al layers as a function of the etching time. The shown values are the averages of $\sigma_{r}$ and $\ell_{c}$ for five different AFM scans. As we can see from the figure, $\sigma_{r}$ and $\ell_{c}$ are proportional to each other, which indicates that the depths of the craters (related to $\sigma_{r}$ ) and the width of the craters (related to $\ell_{c}$ ) grow at proportional rates during etching.

Onto the TCO we deposited a microcrystalline hydrogenated silicon ( $\mu \mathrm{c}-\mathrm{Si}: \mathrm{H})$ p-layer, an amorphous hydrogenated silicon carbide $(\mathrm{a}-\mathrm{SiC}: \mathrm{H})$ p-layer, an a-SiC:H buffer layer, an amorphous hydrogenated silicon (a-Si:H) i-layer, and an a-Si:H n-layer. Finally, we deposited silver, chromium and aluminium layers on the back using a mask with square openings with $0.16 \mathrm{~cm}^{2}$ area. The Ag layer acts as optical back reflector. Together with the $\mathrm{Cr}$ and the $\mathrm{Al}$ layers, it forms the electric back contact. As last processing step, the solar cells were annealed for $30 \mathrm{~min}$ at $130^{\circ} \mathrm{C}$. The solar cells were deposited onto stripes with $2 \times 10 \mathrm{~cm}^{2}$ area. Each stripe contained 30 solar cells.
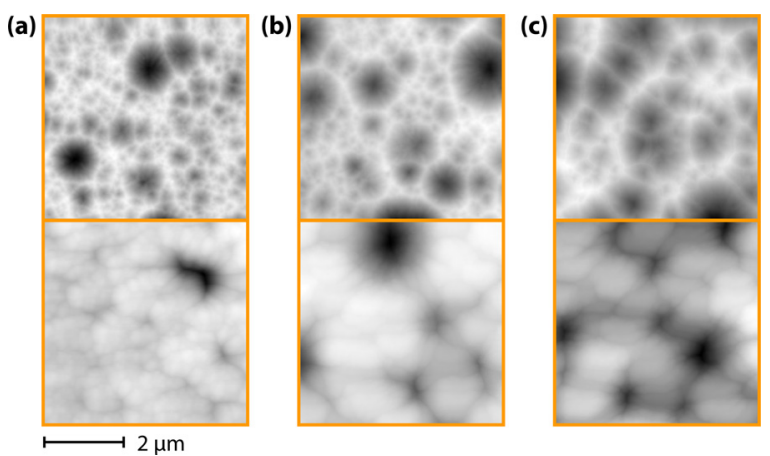

FIG. 7. Atomic force microscopy scans of $\mathrm{ZnO}: \mathrm{Al}$ etched in $\mathrm{HCl}$ of $0.5 \%$ mass fraction (top) and of the back of p-i-n structures deposited on the $\mathrm{ZnO}: \mathrm{Al}$ (bottom). The etch times were $15 \mathrm{~s}$ (a), $30 \mathrm{~s}$ (b), and $45 \mathrm{~s}$ (c). The scans consist of $256 \times 256$ points and are $5 \times 5 \mu \mathrm{m}^{2}$ large.

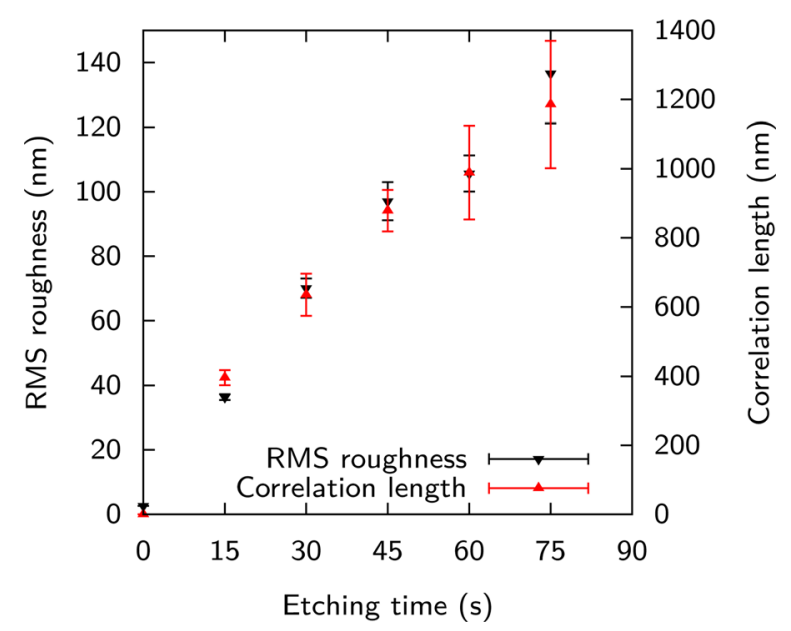

FIG. 8. The RMS roughness and correlation length of the etched $\mathrm{ZnO}: \mathrm{Al}$ layers onto that the solar cells were deposited.

Table II and Fig. 9 show the average external parameters of the 10 best cells of each stripe. They were determined with a Pasan IIc sun simulator/cell tester setup. We observe that the first four stripes (flat and with $\sigma_{r} \approx 35,70,95 \mathrm{~nm}$ ) show a very stable open circuit voltage $\left(V_{\text {oc }}\right)$ and fill factor $(F F)$, while the short circuit current density $\left(J_{\mathrm{sc}}\right)$ and thus the efficiency are increasing with the roughness. The two stripes with the highest $\sigma_{r}$ have lowest efficiency compared to the first four stripes. Since the first four stripes form a very stable set of samples with different surface morphologies, we will use those for validating the combination of the new scattering model with ASA.

\section{B. Simulating solar cells with ASA}

In this section, we discuss and validate the combination of the scattering model with the ASA opto-electric device simulator. For the simulations of the solar cells, we used a layer structure as in Fig. 6. We assumed two scattering interfaces: The interface of $\mathrm{ZnO}: \mathrm{Al}$ with the $\mu \mathrm{c}-\mathrm{Si}: \mathrm{H}$ p-layer and the interface of the a-Si:H n-layer with the back metal. In our simulations, we tested two cases for the morphology of the Si-metal interface: First, we used the accurate morphology obtained from an AFM scan of the back of the p-i-n structure as input. Second, we assumed that both scattering interfaces at either side have the same morphology, i.e., the morphology of the $\mathrm{ZnO}: \mathrm{Al}$. We used scans of $20 \times 20 \mu \mathrm{m}^{2}$ as input for the scattering model.

TABLE II. The external parameters of the deposited solar cells in dependence of the RMS roughness of their front TCO layers. The parameters were measured with a Pasan IIc sun simulator/solar tester setup and averaged on the best 10 cells of stripes with 30 cells. The solar cell area was $0.16 \mathrm{~cm}^{2}$.

\begin{tabular}{lcccc}
\hline \hline$\sigma_{r}(\mathrm{~nm})$ & $V_{\text {oc }}(\mathrm{mV})$ & $J_{\mathrm{sc}}\left(\mathrm{mA} / \mathrm{cm}^{2}\right)$ & $F F(\%)$ & Efficiency $(\%)$ \\
\hline Flat $(2)$ & 885 & 14.4 & 60.6 & 7.77 \\
35 & 888 & 16.3 & 63.9 & 9.25 \\
70 & 887 & 16.9 & 62.6 & 9.42 \\
95 & 887 & 17.1 & 63.6 & 9.64 \\
105 & 866 & 14.5 & 59.3 & 7.46 \\
135 & 869 & 14.6 & 59.1 & 7.50 \\
\hline \hline
\end{tabular}




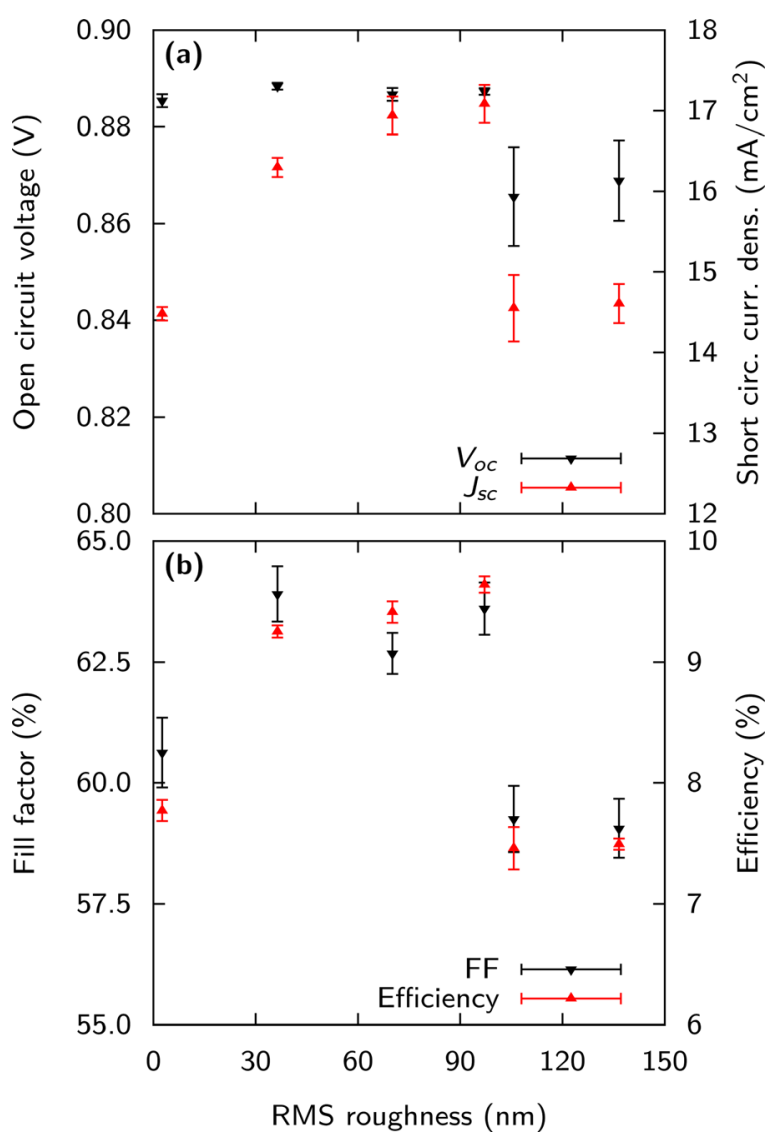

FIG. 9. The external parameters of the deposited solar cells in dependence of the RMS roughness of their front TCO layers. The parameters were measured with a Pasan IIc sun simulator/solar tester setup and averaged on the best 10 cells of stripes with 30 cells. The solar cell area was $0.16 \mathrm{~cm}^{2}$.

To obtain the generation profile with ASA, we used the GENPRO 3 module. In GENPRO 3, light that hits a scattering interface is split into a specular coherent component and a scattered incoherent component, according to the haze. The specular component then is treated according to coherent thin-film optics and thus also predicts interference. Since the scattered light is treated incoherently, GENPRO 3 is dealing with partially coherent light.

We found an offset between the $J_{\mathrm{sc}}$ values obtained from the measured external quantum efficiency (EQE) and from the $J-V$ measurement with the solar simulator. Since for calculating $J_{\text {sc }}$ from the EQE the AM 1.5 spectrum is needed, just as for determining $J_{\mathrm{sc}}$ in ASA, we decided to scale the measured $J-V$ curves with a factor $\beta=J_{\mathrm{sc}}(\mathrm{EQE}) / J_{\mathrm{sc}}(J-V)$ before comparing them to the ASA results. Due to a calibration error for the EQE measurement of the flat cell, the EQE was out of scale by a constant factor over the whole spectrum. We scaled the $J-V$ curve with $\bar{\beta}$, i.e., the mean of the $\beta$ values of the other solar cells. We further scaled the EQE of the flat cell such that $J_{\mathrm{sc}}(\mathrm{EQE})=\bar{\beta} J_{\mathrm{sc}}(J-V)$ was satisfied.

Figure 10 shows the simulated and measured EQE and $J-V$ characteristics of the flat cell (a), the cell deposited onto $\mathrm{ZnO}: \mathrm{Al}$ with moderate roughness $\left(\sigma_{r} \approx 35 \mathrm{~nm}\right)(\mathrm{b})$, and the cell with high roughness $\left(\sigma_{r} \approx 95 \mathrm{~nm}\right)(\mathrm{c})$. In the flat cell, scattering can be neglected. Thus, it can be assumed that thin-film optics is sufficient for calculating the absorption profile. We therefore used the flat cell for the calibration of
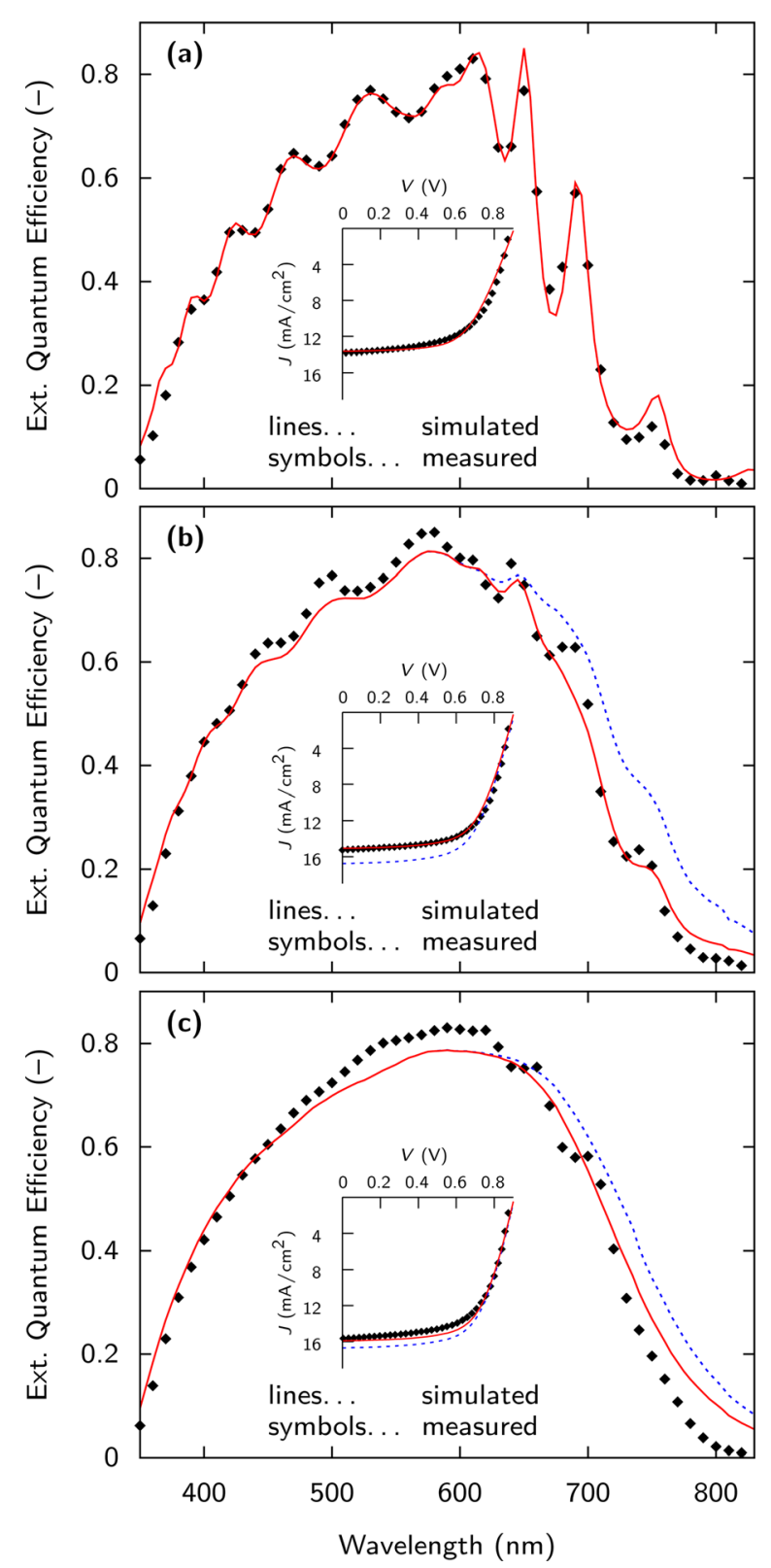

FIG. 10. The results of the simulations with ASA for the cell with flat interfaces (a), with $\sigma_{r} \approx 35 \mathrm{~nm}$ (b) and with $\sigma_{r} \approx 95 \mathrm{~nm}$ (c). The large figures show the EQE while the insets show the $J-V$ characteristics. The red full lines in (b) and (c) show results of simulations where the accurate morphology of the Si-metal interface was used. For the simulations represented by the blue dashed lines, it was assumed that the Si-metal interface has the same morphology as the TCO-Si interface.

the electric parameters in order to obtain a good fit between the simulations and the measurements. Thereafter, we calibrated the thicknesses of the different layers. While the TCO thickness determines the interference fringes up to approximately $600 \mathrm{~nm}$, the i-layer thickness determines the interference fringes for longer wavelengths, where the Si becomes transparent. The thickness of the p-layer can be used to calibrate the EQE in the blue, since this layer absorbs a significant amount of the blue light. The results of the calibrated simulations for the flat cell are shown in Fig. 10(a). The fits for both the EQE and the $J-V$ characteristics are very good. After calibrating ASA for the flat cell, the cells with nanotextured interfaces could be simulated straightforwardly. We 
introduced two scattering layers into the solar cell as described at the beginning of this section. We used the same electrical parameters and layer thicknesses as for the flat cells, only the TCO thickness and the series resistance, which mainly controls the fill factor, had to be calibrated for each cell. To take the AR effect of the rough interfaces into account, we included a $1 \mathrm{~nm}$ thick nonabsorptive effective layer with constant refractive index $n=3$ between the TCO layer and the first p-layer. A summary of all used layers and their thicknesses is given in Table III.

As can be seen in Figs. 10(b) and 10(c), the fits for the rough cells are very satisfying when we use accurate interface morphologies for both scattering interfaces (solid red lines). In (b), the intensity of the interference fringes is slightly underestimated by the simulations. In (c), the simulated EQE slightly underestimates the measured EQE between $450 \mathrm{~nm}$ and $600 \mathrm{~nm}$ and overestimates it above $700 \mathrm{~nm}$. One reason for the shortcomings seen in (c) is the assumption that the solar cell consists of flat layers with rough interfaces that scatter the light. However, the maximal feature height of the $95 \mathrm{~nm} \mathrm{ZnO:Al} \mathrm{is} \mathrm{approx.} 800 \mathrm{~nm}$ making the applicability of this flat-layer approach doubtful.

The intensity of the interference fringes above approximately $600 \mathrm{~nm}$ is strongly underestimated in both (b) and (c). Since the haze decreases with increasing wavelength, one would expect that the intensity of the fringes increases with increasing wavelength. This indeed can be observed at the measured EQE, while it obviously is not the case for the simulated data. The scattering model itself predicts the trend of the haze correctly, as we have seen in Fig. 4. However, above $600 \mathrm{~nm}$ light that is reflected from the (textured) $\mathrm{Si}$ metal interface at the back becomes important. The presence of two scattering interfaces leads to increased complexity. GENPRO 3 does not take into account that the two scattering interfaces have correlated morphologies. In general, the shape of the EQE is predicted well for all three cases shown. The scattering model therefore allows predicting the influence of nano-textured interfaces on the performance of thinfilm silicon solar cells.

The blue dashed lines in Figs. 10(b) and 10(c) show the simulation results when one assumes that the interface morphologies at the TCO-Si and at the Si-metal interface are the same. Since in this case the smoothing effect of the p-i-n layers

TABLE III. The used layers and their thicknesses for the ASA simulations. The first row characterizes the three simulated cells by their TCO morphology. All values are in $\mathrm{nm}$.

\begin{tabular}{lccc}
\hline \hline Morphology & Flat & $\sigma_{r} \approx 35$ & $\sigma_{r} \approx 95$ \\
\hline Glass & $10^{6}$ & $10^{6}$ & $10^{6}$ \\
ZnO:Al & 900 & 840 & 650 \\
Effective AR layer & - & 1 & 1 \\
$\mu$ c-Si:H p-layer & 6 & 6 & 6 \\
a-SiC:H p-layer & 8 & 8 & 8 \\
a-SiC:H buffer layer & 5 & 5 & 5 \\
a-Si:H i-layer & 744 & 744 & 744 \\
a-Si:H n-layer & 20 & 20 & 20 \\
Effective Al like layer & 1 & 1 & 1 \\
Silver back reflector & 300 & 300 & 300 \\
\hline \hline
\end{tabular}

on the morphology is neglected, the scattering is too high resulting in an overestimated EQE above $600 \mathrm{~nm}$. We observed (not shown) that one can get a good fit in the red when adding a $1 \mathrm{~nm}$ thick layer with the optical properties of aluminium between the n-layer and the silver back reflector. Several authors used such a layer and argued that the reflectivity of interfaces between n-type a-Si:H and $\mathrm{Ag}$ is lower than expected from the Fresnel equations. ${ }^{7,27}$ It seems that in these papers the overestimated scattering at the back was compensated by artificially reducing the reflectance of the Si-metal interface.

In this article, we validated the scattering model on solar cells with a $700 \mathrm{~nm}$ thick i-layer. We used a thick i-layer because the far field approach that was used to develop the scattering model is more justified for thicker i-layers. For thinner i-layers with a thickness of $250 \mathrm{~nm}$ to $300 \mathrm{~nm}$, as they are used in state-of-the-art solar cells, also near field optics may be important for modeling the optics in the solar cell accurately. ${ }^{26}$ Further, for cells with thin i-layers, the applicability of the flatlayer approach is even less justified than for the case of the $700 \mathrm{~nm}$ cell studied in this work. Even though the partial destruction of the coherence of the light will be predicted well by the scattering model when it is combined with GENPRO 3, simulating solar cells with thin i-layers remains a complex topic. We, however, expect that the achievements presented in this work are well applicable to microcrystalline solar cells, which contain i-layers with thicknesses in the order of micrometres.

\section{CONCLUSIONS}

Recent developments in scattering models for nanotextured interfaces, as they are present in thin-film silicon solar cells, allow estimating the angular intensity distribution and the haze for both transmission and reflection. In this work, we introduced a full scattering model for nanostructured interfaces as they are present in thin film silicon solar cells. The model is based on the scalar scattering theory. We further evaluated the model for transmission and especially for reflection. We observe good agreement between the simulated and measured $\mathrm{AID}_{R}$ for different TCO-material interfaces. The model therefore can satisfactorily predict the scattering parameters of rough interfaces between arbitrary materials. Further, we combined such a scattering model with the opto-electric ASA simulation software and tested it by simulating and measuring the external parameters and the external quantum efficiency of solar cells with different surface morphologies. This test showed that the scattering model is able to predict the influence of the nano-textured interfaces on the solar cell performance. The results presented in this contribution underline that scattering models based on the scalar scattering theory are powerful tools that can be used for optimizing the morphologies of nano-textured interfaces.

\section{ACKNOWLEDGMENTS}

The work presented in this contribution was funded by Nuon Helianthos. We acknowledge the people from the PVLAB (École polytechniqye fédérale de Lausanne, Switzerland) for providing us with the $\mathrm{ZnO}: \mathrm{B}$ samples and their $n, k$ data. 
We would like to thank Johan van der Cingel (DIMES, Delft University of Technology) and Emile van der Drift and Marc Zuiddam (Kavlilab, Delft University of Technology) for helping us with the AFM measurements. We also acknowledge Mark Workum, Rudi Santbergen, and Bas Vet from the PVMD group for supporting us with the ASA simulations and for the useful discussions.

${ }^{1}$ D. L. Staebler and C. R. Wronski, Appl. Phys. Lett. 31, 292 (1977).

${ }^{2}$ H. W. Deckman, C. R. Wronski, H. Witzke, and E. Yablonovitch, Appl. Phys. Lett. 42, 968 (1983).

${ }^{3}$ M. Berginski, J. Hüpkes, W. Reetz, B. Rech, and M. Wuttig, Thin Solid Films 516, 5836 (2007).

${ }^{4}$ K. Jäger and M. Zeman, Appl. Phys. Lett. 95, 171108 (2009).

${ }^{5}$ D. Dominé, F.-J. Haug, C. Battaglia, and C. Ballif, J. Appl. Phys. 107, 044504 (2010).

${ }^{6}$ K. Bittkau, M. Schulte, T. Beckers, and R. Carius, Proc. SPIE 7725, $77250 \mathrm{~N}(2010)$.

${ }^{7}$ M. Zeman, R. A. C. M. M. van Swaaij, J. W. Metselaar, and R. E. I. Schropp, J. Appl. Phys. 88, 6436 (2000).

${ }^{8}$ J. Krč, M. Zeman, F. Smole, and M. Topič, J. Appl. Phys. 92, 749 (2002).

${ }^{9}$ J. Krč, M. Zeman, O. Kluth, F. Smole, and M. Topič, Thin Solid Films 426, 296 (2003).

${ }^{10}$ J. Springer, A. Poruba, and M. Vanecek, J. Appl. Phys. 96, 5329 (2004).

${ }^{11}$ M. Schulte, K. Bittkau, K. Jäger, M. Ermes, M. Zeman, and B. E. Pieters, Appl. Phys. Lett. 99, 111107 (2011).

${ }^{12}$ K. Yee, IEEE Trans. Antennas Propag. 14, 302 (1966).
${ }^{13}$ A. Taflove and S. C. Hagness, Computational Electrodynamics: The FiniteDifference Time-Domain Method, 3rd ed. (Artech House Inc., 2005).

${ }^{14} \mathrm{~J}$. Jin, The Finite Element Method in Electromagnetics (Wiley, 2002).

${ }^{15} \mathrm{M}$. Born and E. Wolf, Principles of Optics, 7th ed. (Cambridge University Press, 1999), pp. xxxiv +952 .

${ }^{16}$ J. E. Harvey, C. L. Vernold, A. Krywonos, and P. L. Thompson, Appl. Opt. 38, 6469 (1999).

${ }^{17}$ M. Plancherel and M. Leffler, Rend. Circ. Mat. Palermo 30, 289 (1910).

${ }^{18}$ K. Sato, Y. Gotoh, Y. Wakayama, Y. Hayashi, K. Adachi, and N. Nishimura, Rep. Res. Lab., Asahi Glass Co. Ltd. 42, 129 (1992).

${ }^{19}$ O. Kluth, B. Rech, L. Houben, S. Wieder, G. Schöpe, C. Beneking, H. Wagner, A. Löffl, and H. Schock, Thin Solid Films 351, 247 (1999).

${ }^{20}$ D. Dominé, P. Buehlmann, J. Bailat, A. Billet, A. Feltrin, and C. Ballif, Phys. Status Solidi (RRL) 2, 163 (2008).

${ }^{21} \mathrm{P}$. Klapetek, "Characterization of randomly rough surfaces in nanometric scale using methods of modern metrology," Ph.D. dissertation (Masaryk University Brno, 2003).

${ }^{22}$ K. Jäger, O. Isabella, R. A. C. M. M. van Swaaij, and M. Zeman, Meas. Sci. Technol. 22, 105601 (2011).

${ }^{23}$ J. Sap, O. Isabella, K. Jäger, and M. Zeman, Thin Solid Films 520, 1096 (2011).

${ }^{24}$ T. Markvart, J. Opt. A, Pure Appl. Opt. 10, 015008 (2008).

${ }^{25}$ B. Vet, B. Grancic, O. Isabella, S. Solntsev, and M. Zeman, in Proceedings of the 24th European Photovoltaic Solar Energy Conference (2009), p. 2682.

${ }^{26}$ K. Bittkau and T. Beckers, Phys. Status Solidi A 207, 661 (2010).

${ }^{27}$ H. Stiebig, A. Kreisel, K. Winz, N. Schultz, C. Beneking, T. Eickhoff, H. Wagner, and M. Meer, in 1994 IEEE First World Conference on Photovoltaic Energy Conversion (1994), Vol. 1, p. 603. 